\title{
ERS International Congress 2020 Virtual: highlights from the Allied Respiratory Professionals Assembly
}

\author{
Elizabeth Smith ${ }^{1,11}$, Max Thomas ${ }^{2,11}$, Ebru Calik-Kutukcu $\mathbb{1}^{3,11}$, \\ Irene Torres-Sánchez (10,11, Maria Granados-Santiago (10 ${ }^{5,11}$, \\ Juan Carlos Quijano-Campos $\mathbb{1}^{6,11}$, Karl Sylvester ${ }^{7}$, Chris Burtin $^{8}$, \\ Andreja Sajnic $\mathbb{1}^{9}$, Jana De Brandt ${ }^{8}$ and Joana Cruz (ib ${ }^{10}$
}

\begin{abstract}
Affiliations: ${ }^{1}$ Children's Lung Health, Wal-Yan Respiratory Centre, Telethon Kids Institute, Perth, Australia. ${ }^{2}$ Cardiopulmonary Exercise Testing Service, University Hospitals Birmingham, Birmingham, UK. ${ }^{3}$ Faculty of Physical Therapy and Rehabilitation, Hacettepe University, Ankara, Turkey. ${ }^{4}$ Dept of Physical Therapy, Faculty of Health Sciences, University of Granada, Granada, Spain. ${ }^{5}$ Dept of Nursing, Faculty of Health Sciences, University of Granada, Granada, Spain. ${ }^{6}$ Research and Development, Royal Papworth Hospital NHS Foundation Trust, Cambridge Biomedical Campus, Cambridge, UK. ${ }^{7}$ Respiratory Physiology, Royal Papworth and Cambridge University Hospitals NHS Foundation Trusts, Cambridge, UK. ${ }^{8}$ REVAL - Rehabilitation Research Center, BIOMED - Biomedical Research Institute, Faculty of Rehabilitation Sciences, Hasselt University, Diepenbeek, Belgium. ${ }^{9}$ Dept for Respiratory Diseases Jordanovac, University Hospital Center, Zagreb, Croatia. ${ }^{10}$ Center for Innovative Care and Health Technology (ciTechCare), School of Health Sciences (ESSLei), Polytechnic of Leiria, Leiria, Portugal. ${ }^{11}$ These authors contributed equally.
\end{abstract}

Correspondence: Joana Cruz, Center for Innovative Care and Health Technology (ciTechCare), School of Health Sciences (ESSLei), Polytechnic of Leiria, Campus 2, Morro do Lena - Alto do Vieiro, Apartado 4163, 2411-901 Leiria, Portugal. E-mail: joana.cruzQipleiria.pt

ABSTRACT This article provides an overview of outstanding sessions that were (co)organised by the Allied Respiratory Professionals Assembly during the European Respiratory Society International Congress 2020, which this year assumed a virtual format. The content of the sessions was mainly targeted at allied respiratory professionals, including respiratory function technologists and scientists, physiotherapists, and nurses. Short take-home messages related to spirometry and exercise testing are provided, highlighting the importance of quality control. The need for quality improvement in sleep interventions is underlined as it may enhance patient outcomes and the working capacity of healthcare services. The promising role of digital health in chronic disease management is discussed, with emphasis on the value of end-user participation in the development of these technologies. Evidence on the effectiveness of airway clearance techniques in chronic respiratory conditions is provided along with the rationale for its use and challenges to be addressed in future research. The importance of assessing, preventing and reversing frailty in respiratory patients is discussed, with a clear focus on exercise-based interventions. Research on the impact of disease-specific fear and anxiety on patient outcomes draws attention to the need for early assessment and intervention. Finally, advances in nursing care related to treatment adherence, self-management and patients' perspectives in asthma and chronic obstructive pulmonary disease are provided, highlighting the need for patient engagement and shared decision making. This highlights article provides readers with valuable insight into the latest scientific data and emerging areas affecting clinical practice of allied respiratory professionals.

@ERSpublications

This article provides an overview of outstanding sessions that were (co)organised by @ERS_Assembly9 during \#ERSCongress 2020 Virtual https://bit.ly/2IwZNEI

Cite this article as: Smith E, Thomas M, Calik-Kutukcu E, et al. ERS International Congress 2020 Virtual: highlights from the Allied Respiratory Professionals Assembly. ERJ Open Res 2021; 7: 008082020 [https://doi.org/10.1183/23120541.00808-2020]. 


\section{Introduction}

The virtual European Respiratory Society (ERS) International Congress 2020 offered allied respiratory professionals a wide range of appealing sessions (co)organised by Assembly 9 (Allied Respiratory Professionals), including symposia, Expert View, Hot Topics, Clinical Year in Review and 266 abstract sessions spread over four oral communication and 13 e-poster sessions.

Early Career Members of the Allied Respiratory Professionals Assembly, including respiratory function technologists and scientists (E. Smith and M. Thomas), physiotherapists (E. Calik-Kutukcu and I. Torres-Sánchez), and nurses (M. Granados-Santiago and J.C. Quijano-Campos), were invited to report on the following sessions selected by group chairs of the Assembly, which focused on recent research advances: two oral presentation sessions on the quality improvements in lung function and sleep diagnostics, and novel insights into nursing interventions for managing patients with asthma and chronic obstructive pulmonary disease (COPD); three symposia on digital health, managing frailty in patients with acute or chronic lung disorders and psychological wellbeing in chronic lung diseases; and an Expert View in physiotherapy in chronic respiratory infections with a focus on airway clearance techniques. We summarised the latest scientific and clinical insights gained from each session, targeting delegates who were present in the virtual sessions as well as those unable to attend.

\section{Group 9.01: Respiratory Function Technologists and Scientists}

\section{Oral presentation session: Quality improvements in lung function and sleep diagnostics}

This oral presentation session included the highest scored abstracts submitted to the Respiratory Function Technologists and Scientists (9.01) group.

Quality improvements in a sleep clinic: to increase capacity and time to treat in patients with obstructive sleep apnoea (P. Coss, Ireland)

P. Coss described the outcome of a quality improvement programme in the Sleep Dept at St James's Hospital (Dublin, Ireland). This sought to reduce the time to treatment from a pre-intervention of 32 weeks to 9 weeks and address inefficiencies to increase the capacity of the department. Pareto analysis showed that the most significant delay was caused by general practitioner referral to the first appointment with the sleep consultant. Through a series of Plan-Do-Study-Act (PDSA) cycles, the team identified those interventions which added value, which included adopting a Proforma for assessing the sleepy patient, and co-locating consultant and physiologist assessments. Following the interventions, attendance was shown to be both improved and more consistent. The time from initial intervention to review in the sleep clinic was reduced from 196 to 14 days and the number of new patients seen per month increased from four to 15. This talk was an excellent example of how standard quality improvement processes can be implemented in routine practice to achieve significant patient benefits.

\section{Early effect of continuous positive airway pressure therapy on right ventricular function in patients with newly diagnosed obstructive sleep apnoea (P. Coss, Ireland)}

The second of piece of work presented by P. Coss was part of a cross-departmental project built on previous findings [1] and analysed right ventricular function in 19 obstructive sleep apnoea (OSA) patients (70\% male) following a 12-week intervention of continuous positive airway pressure (CPAP) treatment (mean compliance $4.1 \mathrm{~h} \cdot$ night $^{-1}$ ). Outcome measures included basal right ventricular diameter, tricuspid annular plane systolic excursion (TAPSE) and fractional area change (FAC), the latter two being measures of global right ventricular function. Following CPAP treatment, a small but significant reduction in right ventricular size, and an increase in FAC and TAPSE, i.e. markers of improved right ventricular ejection, was observed. It was proposed that, in patients with OSA, there are likely subclinical changes in the structure and function of the heart which are, at least in part, reversible with the application of CPAP treatment. The presented results are preliminary and it will be exciting to see the results of the study once completed.

\section{Quality control of cardiopulmonary exercise equipment (T. Souren, Belgium)}

T. Souren presented an observation of the performance of four metabolic carts in use at University Hospital Antwerp (Antwerp, Belgium). The team used the Metabolic Simulator (Relitech Systems, Nijkerk, The Netherlands) to verify the performance of each cart at four different metabolic levels, with breathing frequencies from 20 to 80 breaths. $\mathrm{min}^{-1}$, and oxygen uptake $\left(V_{\mathrm{O}_{2}}^{\prime}\right)$ and carbon dioxide production $\left(V^{\prime} \mathrm{CO}_{2}\right)$ from 1 to $4 \mathrm{~L} \cdot \mathrm{min}^{-1}$. The carts studied included the Metalyser II (Cortex, Leipzig, Germany), Powercube (Ganshorn, Niederlauer, Germany), CPET Oxygen Sensor II (Geratherm Respiratory, Bad Kissingen, Germany) and Vyntus CPX (Vyaire, Mettawa, IL, USA). Varying performance of the four devices was demonstrated, with all devices being outside of the acceptability limits at least once (3\% for $V^{\prime} \mathrm{O}_{2}$ and $V^{\prime} \mathrm{CO}_{2}, 2 \%$ for minute ventilation $\left(V_{\mathrm{E}}^{\prime}\right)$, and 0.04 absolute for respiratory exchange ratio (RER)). It is 
advocated that a metabolic simulator should be part of at least a once-yearly routine quality check of metabolic carts and used as a complementary practice to the use of biological controls, to understand equipment variability and impacts on future data collection.

\section{A RER of 1.05 should not be used to determine maximal effort during cardiopulmonary exercise testing (M. Thomas, UK)}

M. Thomas presented a review of the American Thoracic Society (ATS)/American College of Chest Physicians [2] and ERS [3] recommendations for determining maximal effort during cardiopulmonary exercise testing (CPET) and noted that both publications quoted target RER values $(>1.15$ and $>1.05$, respectively). A retrospective review of CPET data collected at University Hospital Birmingham (Birmingham, UK), where the patient achieved an RER $>1.15(\mathrm{n}=176)$ to determine CPET outcomes at RERs of 1.05 and 1.15, compared with volitional exhaustion, was performed. From the patients assessed for pre-operative risk, at volitional exhaustion, $25 \%$ were classified as high risk $\left(V_{\mathrm{O}_{2}}^{\prime}<15 \mathrm{~mL} \cdot \mathrm{min} \cdot \mathrm{kg}^{-1}\right)$, whereas at $\mathrm{RER}=1.05$ and $\mathrm{RER}=1.15,77 \%$ and $45 \%$ of patients were considered high risk, respectively. In a subanalysis of patients investigated for unknown cause of breathlessness, the proportion of those who returned an abnormal result based on ERS criteria [3] was $63 \%, 75 \%$ and $97 \%$ at volitional exhaustion, $\mathrm{RER}=1.15$ and $\mathrm{RER}=1.05$, respectively. The team provided robust evidence that a pre-determined RER value should not be used to determine the end of test as this is likely to overestimate pre-operative risk and result in mismanagement of patients undergoing CPET for diagnostic purposes.

\section{A comparison of three sets of paediatric reference values for CPET using cycle ergometry}

(P. Burns, UK)

P. Burns from the Royal Hospital for Children (Glasgow, UK) presented percent predicted $V^{\prime} \mathrm{O}_{2}$ peak $(\mathrm{n}=766)$, where predicted values were calculated using three commonly used reference equations from CoOper et al. [4], Blanchard et al. [5] and Bongers et al. [6]. Bland-Altman analysis demonstrated wide limits of agreement (LoA) between all three equations, which was the greatest for Cooper versus Bongers (upper LoA 48\%, lower LoA -26\%). It was noted that the commonly used Cooper equation was derived from the smallest dataset $(n=109)$ and simplest data analysis but the largest age range $(6-17$ years), and while the Blanchard equation looks initially promising with the largest dataset and multivariate analysis, it was limited in its use in a paediatric laboratory due to the age range of subjects included (12-18 years). Findings evidenced that the reference equation used will have pronounced implications on the interpretation of paediatric CPET data, and that a "Global Lung Function Initiative" (GLI) approach of all age reference equations for CPET is needed to standardise laboratory practice and advance the field.

Deep learning automates complete quality control of spirometric manoeuvres (N. Das, Belgium) N. Das, a PhD candidate from Leuven University (Leuven, Belgium), presented one study on the use of deep learning for the purpose of spirometry quality control. The model presented was built upon previous work [7], further including the criteria of tidal breathing and maximal inspiration before and after forced expiration. Using the sample dataset, the convolutional neural network model had a greater area under the receiver operating characteristic curve $(0.91)$, accuracy $(87 \%)$ and specificity $(81 \%)$ than the technician ATS/ERS rule-based model (0.77, 67\% and 32\%, respectively) [8]. The major strength identified, aside from the accuracy, was that the Shapley values were able to provide a clear visual feedback as to why the manoeuvre had failed the quality control check.

This was an excellent example of how deep learning could be used to complement the visual cognition of skilled technicians, working towards the automatic definition of spirometric acceptability. The authors of the study are currently looking to adapt the tool to accommodate the latest 2019 guidelines [9].

Differences between percent predicted and z-score: the impact of severity classification in spirometry (A. Silva, Portugal)

The final presentation in this session, from A. Silva, highlighted that the severity criteria used can affect the classification of disease in a paediatric population. The 2012 GLI reference equations [10] were used to generate percent predicted and z-scores for 1869 children (aged 3-18 years, 55\% male) who attended Santa Maria Hospital (Lisbon, Portugal). Normality and severity classifications were defined according to both the 2005 ATS/ERS criteria [11] and the more recent 2014 proposal by QUANJER et al. [12] (table 1). 19\% of the spirometry tests returned a different severity classification using the two methods described. Using QUANJER et al. [12] criteria, 27\% (120 out of 440) of mild (ATS/ERS) cases were classified as normal. Notably, almost all ATS/ERS definitions of moderate (77 out of 84), moderate to severe (44 out of 44) and severe (39 out of 41 ) cases increased by $\sim 1$ severity score when reanalysed using Quanjer's z-score model. While it was agreed that the new classification system is simple and easy to implement, it was also acknowledged that this would affect severity classification in some children which requires clinical 
TABLE 1 Recommendations for the classification of airway obstruction severity, as defined by the 2005 American Thoracic Society/European Respiratory Society guidelines [11] (\% pred) and the more recent 2014 proposal by QUANJER et al. [12] (z-score)

Classification

$\begin{array}{lc} & \% \text { pred } \\ \text { Mild } & \geqslant 70 \\ \text { Moderate } & 60-69 \\ \text { Moderate to severe } & 50-59 \\ \text { Severe } & 35-49 \\ \text { Very severe } & <35\end{array}$

\section{Forced expiratory volume in $1 \mathrm{~s}$}

z-score

$\geqslant-2.0$

$-2.5--2.0$

$-3.0--2.5$

$-4.0--3.0$

$<-4.0$

validation. It should, however, be noted that clinical validation of the ATS/ERS severity scoring system is not yet robust.

Take-home messages

- There exist multiple tools for interpreting respiratory function data, the choice of which can have profound implications for disease classification. This requires further standardisation across the field.

- Internal quality improvement plans can be effective tools to increase the capacity and efficiency of a sleep department.

- CPET should not be terminated at a pre-determined RER but performed to volitional exhaustion, when it is safe to do so.

- A metabolic simulator can be a valuable tool to understand inherent differences between metabolic carts, when used as an adjunct to a biological control programme.

- The automation of spirometry quality control is being advanced by machine learning.

\section{Symposium: Digital health: a brave new world?}

The healthcare sector is being rapidly transformed by the development and innovation of digital technologies. This symposium started by focusing on patients and end-users of these technologies, before focusing on specific applications of digital technologies in the management of asthma; it ended by looking at new horizons in digital technologies in respiratory medicine and discussing the promise of artificial intelligence.

\section{The patient's perspective (D. Hamerlijnck, The Netherlands)}

D. Hamerlijnck is a patient expert with severe asthma. In this presentation, D. Hamerlijnck postulated that the vision for the future digitisation of care seemed to be replacing the doctor with an algorithm based on guidelines [13]. While this is a useful strategy for the majority of patients, these guidelines are based on what is most common in a patient population. D. Hamerlijnck reported to be an example in which these algorithms cause issues, because D. Hamerlijnck is a patient with severe asthma who does not wheeze or cough. The talk then focused on patient advocacy and ensuring that patients and end-users are involved in the development of these digital technologies. The other speakers in this session all dedicated time in their presentations to reinforce the message that patient and end-user involvement is crucial to the future of these technologies.

\section{Digital technology for asthma (O.S. Usmani, UK)}

This presentation addressed the use of digital technologies in the management and diagnosis of asthma. O.S. Usmani suggested the key drivers for digital solutions are prevalence of disease, advancements in technology (both innovation of technology and patient digital literacy) and patient engagement. Development of digital technologies is critical in asthma with 300 million patients worldwide, 50\% of which do not take medications as prescribed and $60 \%$ do not attend follow-ups [14]. Initial focus in the development of these technologies in asthma was on monitoring medication remotely. The aim was to identify those patients poorly controlled and in need of additional attention to help achieve disease control. Innovations employed included digital inhaler trackers [15] and new methods of monitoring device adherence [16]. Sulaiman et al. [17] developed an electronic acoustic recording device for dry powder inhalers to identify the types of error with their use. They found that $48 \%$ of patients would fail to generate a peak inspiratory flow rate $>35 \mathrm{~L} \cdot \mathrm{min}^{-1}$. Other errors identified included patients performing multiple inhalations $(23 \%)$, not inhaling at all despite introducing the blister pack $(7.4 \%)$ or exhaling into 
the device (5.4\%). Smart sensors introduced in inhaler devices demonstrated higher adherence in children aged 6-15 years compared with the same device with the sensor function disabled (84\% versus 30\%) [18]. The use of mobile applications for self-management of asthma was systematically reviewed by Hui et al. [19]. The most successful interventions included multiple features such as symptom monitoring and medication reminders. O.S. Usmani then echoed D. Hamerlijnck's message about the importance of including the patient and end-users in the digital technology development process, and detailed a project called "myaircoach" that involves the analysis, modelling and sensing of both physiological and environmental factors for the customised and predictive self-management of asthma. The group developing the project is making the user the centre of its design and implementation by approaching asthma patients and healthcare practitioners regarding the functionality and use of these systems for self-management [20]. Patients were most interested in technology improving self-monitoring, collecting data to present to healthcare professionals that demonstrates how their asthma has been, and alerting the patients and/or healthcare professionals to a deterioration in their asthma control before they would normally notice. In contrast, healthcare professionals wanted a device that offers advice regarding when additional medical attention should be sought or that provides instructions on how to manage their asthma in an emergency.

Artificial intelligence in respiratory medicine (V. Poberezhets, Ukraine)

V. Poberezhets discussed new horizons in digital technologies for respiratory medicine where an ontological structure of digital medicine was presented (figure 1) [21]. The use of artificial intelligence (AI) and machine learning has been employed to improve diagnostic yield and decrease workload in thoracic imaging $[22,23]$, histopathology and cytology [24, 25]. AI could be used to improve diagnostic accuracy in the interpretation of pulmonary function tests, where it was demonstrated to have a higher degree of accuracy than pulmonologists [26].

V. Poberezhets highlighted interesting future developments in AI such as clinical decision support systems that will aggregate all data obtained in a patient's pathway [27]. The current systems are limited by having to process unstructured data such as patient complaints or observations made in clinical examinations and recorded in notes; AI may address these issues.

\section{Artificial intelligence in clinical trials (K. Kostikas, Greece)}

K. Kostikas's talk detailed the promise of AI in clinical trials and research. The traditional drug development cycle takes up to 15 years and requires up to EUR1.7 billion, and AI may be able to reduce these figures by aiding in clinical trial design, feasibility assessment, patient recruitment, blinding of trials by aggregating anonymous data, performing the literature review, matching patients, real-time monitoring of data during trials, data collection and sharing [28]. AI has theoretical applications to increase study power by reducing population heterogeneity, for prognostic enrichment by selecting patients more likely to have measurable clinical end-points and for predictive enrichment by identifying patients more likely to respond to treatment [29].

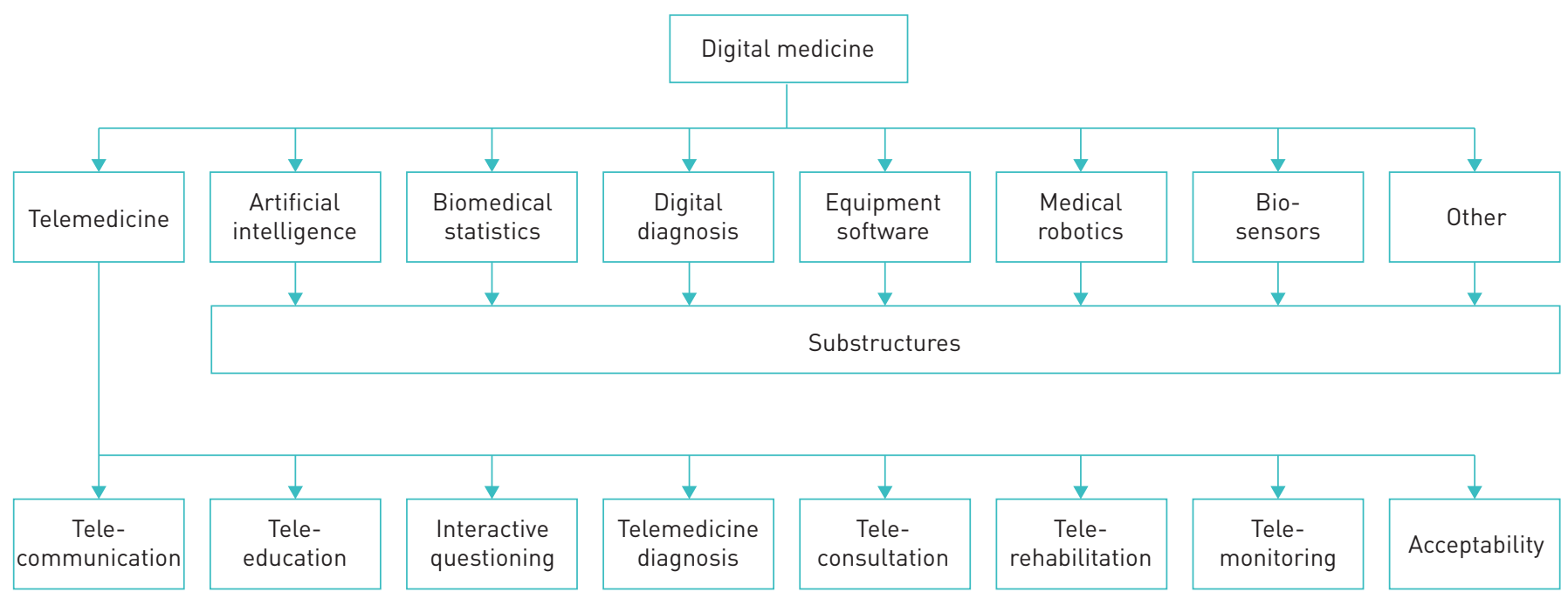

FIGURE 1 The ontological structure of digital medicine. Reproduced from [21] with permission. 
Take-home messages

- Patient's trust in clinicians is, in part, developed by nonverbal communications that can be impaired by remote technologies and tele-consultations. Future technologies should consider this during development.

- The end-users are both patients and clinicians, and both have distinctly different desires and expectations for remote monitoring tools.

- Al is not being developed to replace clinicians and we should embrace it, not fear it: "a computer is not better than a human brain, but a computer supporting the human brain is better than the human brain alone".

\section{Group 9.02: Physiotherapists}

\section{Expert View: Physiotherapy in chronic respiratory infections (A. Spinou, UK)}

According to the PICO (Population, Intervention, Comparison, Outcome) framework [30], A. Spinou presented a detailed overview about the ability of airway clearance techniques to reduce the rate of exacerbations, hospitalisations or improve quality of life in patients with COPD, cystic fibrosis (CF) and bronchiectasis. First, A. Spinou gave some background information about airway clearance techniques and presented airway clearance technique evidence for these three chronic respiratory diseases. A. Spinou then talked about research challenges in this area and the implementation of airway clearance techniques in clinical practice.

Airway clearance is a defence mechanism that is based on the relationship between the mucociliary escalator and cough. Cough is important for effective clearance of secretions in addition to helping to collect thicker secretions and increase secretion volume. Another important factor for removing secretions is airway surface liquid [31]. Airway clearance is compromised in some diseases due to impaired mucociliary function, alterations in secretion production and mucus rheology (i.e. fluid flow and deformation for mucus), and impaired cough reflex as a defence mechanism [32]. The main problem is that patients with bronchiectasis enter a vicious cycle in which impaired mucociliary clearance leads to structural airway damage and consequently lower airway tract infection that leads to inflammation. Inflammation causes recurrent pulmonary infections that increase mucus production. Airway clearance techniques have the potential to break this cycle as increasing mucociliary clearance decreases structural airway damage [33]. Airway clearance techniques are techniques (with or without the use of devices) that have been employed alone and in combination to facilitate airway clearance and increase the effectiveness of cough. Techniques include gravity-assisted positioning/postural drainage, manual techniques like percussion, vibrations, shaking and overpressure, active cycle of breathing techniques (ACBT), autogenic drainage and slow expiration with the glottis opened in the lateral posture (ELTGOL). There are also many devices to assist airway clearance: positive expiratory pressure (PEP), oscillating PEP and high-frequency chest wall oscillation devices [34]. The rationale of these techniques and devices is based on physiological mechanisms including gravity/positioning, mucous viscoelasticity, two-phase gas-liquid interaction, collateral ventilation, interdependence and equal pressure point [35]. Interestingly, some techniques are most preferred in different countries; whereas ACBT is the most preferred method in the UK, PEP devices are frequently used in the USA by physiotherapists treating patients with bronchiectasis [36].

The outcomes most frequently used to assess the effectiveness of airway clearance techniques are sputum volume/weight (dry or wet), mucus transport rate, ventilation scanning, pulmonary function, lung clearance index, oxygen saturation, symptoms like dyspnoea and cough, adverse effects, duration and frequency of exacerbations and hospitalisations, need for antibiotics, health-related quality of life, patient preference, treatment adherence, and mortality [37]. There are few studies that investigate the long-term effect of airway clearance techniques on outcomes. A Cochrane review comparing airway clearance techniques to no airway clearance techniques during an acute exacerbation of COPD reported no change in the number of exacerbations at 6-month follow-up, but it included studies with a small sample size [38]. In patients with stable COPD, there was a reduction in the need for hospital admission but there was no change in the total number of days at the hospital in the long term. Studies showed that airway clearance techniques reduced the need for antibiotics in stable COPD [38]. A multicentre randomised controlled study also showed that the PEP technique was not effective in reducing the number of exacerbations, number of respiratory-related hospitalisations, total number of hospitalised days, need for antibiotics and mortality compared with controls at 6 months of follow-up after an acute exacerbation [39]. This study also demonstrated that there was no benefit in terms of quality of life [39]. In patients with bronchiectasis, a Cochrane review reported an improvement in quality of life but no change in frequency of exacerbations at 3-month follow-up [40]. In a randomised placebo controlled study published in 2018, patients with bronchiectasis that used the ELTGOL technique showed an improvement in 
cough-related quality of life and health-related quality of life and decreased frequency of exacerbations [41]. Several Cochrane reviews have been published regarding airway clearance techniques in CF [33, 41]. Most of them compare the effect of different techniques on mortality. A Cochrane review that included eight studies comparing airway clearance techniques with no airway clearance techniques in CF showed that no data is available regarding number of exacerbations per year, number of days in hospital per year, number of days of intravenous antibiotics per year and death [42].

In conclusion, there was low quality of evidence about the effectiveness of airway clearance techniques in chronic respiratory diseases [43]. The most prevalent methodological issues were difficulties in adherence and compliance in long-term investigations, ethical problems, lack of blinding, poor study design, poorly defined or misinterpreted techniques and lack of selecting appropriate outcome measurements. In a clinical setting, the selection of the optimal airway clearance techniques should take into account the diagnosis, the severity/stage of disease, whether the patient is in a stable or exacerbation phase and existing comorbidities. Physiotherapists should also consider underlying physiology, contraindications/precautions, equipment, cost and burden of treatment technique. Clinicians should also pay attention to preference/ ability, needs, age and treatment adherence of the patients when planning treatment. As a key message, clinical practice about airway clearance techniques is mainly guided by physiology rationale and short-term benefits. There is an urgent need to take action and perform high-quality research on the long-term effectiveness of airway clearance techniques [43].

Take-home messages

- There is low quality of evidence about the effectiveness of airway clearance techniques in chronic respiratory diseases (COPD, bronchiectasis and CF).

- In a clinical setting, the selection of the optimal airway clearance technique should take into account the diagnosis, the severity/stage of disease, whether the patient is in a stable or exacerbation phase and existing comorbidities.

Symposium: Managing frailty in patients with acute or chronic lung disorders

In this symposium, several expert speakers provided an update on the latest knowledge about frailty in respiratory diseases.

Diagnosing, management and measures of frailty (M. Cesari, Italy)

M. Cesari started by explaining that frailty is not a disease. There is a consensus definition of frailty as "a medical syndrome with multiple causes and contributors that is characterised by diminished strength, endurance, and reduced physiologic function that increases an individual's vulnerability for developing increased dependency and/or death" [44]. Many practical operational definitions of frailty are available. In the phenotypic operational definition of frailty provided by FriED et al. [45], a frail individual was defined according to a number of predefined criteria: involuntary weight loss, weakness, poor endurance or exhaustion, slowness and sedentary behaviour. A person is considered frail when meeting at least three criteria and pre-frail when meeting one or two criteria. Another important school of thought tried to propose an operational definition that aimed to provide a measure of the age-related accumulation of deficits that the person presents in order to guess a surrogate of biological ageing [46]. M. Cesari remarked that the more deficits a person presents, the frailer he/she will be [47].

In the literature, we can find a huge number of instruments available to measure frailty. Nine instruments are highly cited (over 200 citations). The most common assessment context was observational studies in older community-dwelling adults [48]. Frailty is the target condition for applying a comprehensive geriatric assessment [49], a multidimensional diagnostic and therapeutic process for dealing with highly complex individuals. M. Cesari noted that all information provided can be found in the "Integrated care for older people" document published by the World Health Organization [50].

\section{Skeletal muscle stem cells and capillaries: important neighbours in muscle tissue of COPD patients (T. Snijders, The Netherlands)}

T. Snijders explained how muscle fibres relate to frailty. Satellite cells, as stem cells, are able to provide growth and regeneration capabilities to the muscle fibre. Satellite cell content and function are known to decline with increasing age [51]. Satellite cell function is even lower in COPD patients compared with age-matched controls and, therefore, it might be a key factor in the accelerated ageing of skeletal muscle tissue [52]. Circulation plays an important role in the activation and functioning of the satellite cell [53]. Hence, it is not surprising to find satellite cells close to capillaries. This distance has been shown to be increased in healthy elderly adults compared with young adults (particularly in type II muscle fibres) [54]. 
Performing prolonged exercise training can restore the balance between satellite cells and capillaries. The preliminary results in the studies reported by $\mathrm{T}$. Snijders and co-workers showed no significant difference in satellite cell content between COPD and age-matched controls. Fibre capillarisation was lower in type I (and type II) muscle fibres compared with young elders as well as age-matched healthy controls.

Most importantly, T. Snijders and co-workers investigated the satellite cell distance to its nearest capillary [54]. In healthy young individuals, they found no differences between type I and type II muscle fibres, while in healthy older adults, this distance was $\sim 20 \%$ higher in type II muscle fibres. In COPD patients, this distance was equally large in type II muscle fibres compared with healthy older adults. In type I muscle fibres, there was a greater distance compared with healthy young and healthy older adults. Satellite cell distance to nearest capillary was greater in type I muscle fibres of COPD versus controls. In conclusion, changes in muscle tissue are associated with frailty and can be modified by exercise training.

\section{Frailty during and after hospitalisation (M. Herridge, Canada)}

M. Herridge spoke about critical illness as a model for frailty, functional and cognitive disability, and the intersection of these. In the study by BAGSHAw et al. [55], the association between frailty and short- and long-term outcomes among critically ill patients was shown. They found that frailty was common among critically ill adults aged $\geqslant 50$ years, and identified a population at increased risk of adverse events, morbidity and mortality. Frailty can be modifiable over time as patients showed that they may transition to a better frailty state. Disability and cognitive impairment may overlap with frailty, but many patients acquire frailty in isolation [56].

After a severe episode of acute respiratory distress syndrome (ARDS), patients sustain important muscle wasting and weakness of $\sim 20 \%$ of their baseline weight [57].

Levine et al. [58] and Puthucheary et al. [59] showed the relentless atrophy of the diaphragm and muscles of the axial skeleton, respectively. Among older persons with critical illness, more than half died within 1 month or experienced significant functional decline in the following year, with particularly poor outcomes in those who had high levels of pre-morbid disability. These results may help to inform discussions about the prognosis and goals of care before and during critical illness [60]. However, it is not just physical disability but also cognitive disability. Hopkins et al. [61] showed a decrement in processing speed, memory, executive function, poor attention and concentration in survivors of severe ARDS. Critical patients may be vulnerable to long-term cognitive dysfunction [62]. Patients can also show mood disorders, such as persecutory delusion and post-traumatic stress disorder [63].

Frailty in chronic lung disorders (L. Lahousse, Belgium)

In this presentation, L. Lahousse discussed three main points: 1) the prevalence of frailty in COPD patients, 2) the mechanisms that frailty and COPD share, and 3) the impact of frailty in patients with COPD. Frailty is higher in these patients (10.2\%) compared with people without COPD (3.4\%) and its prevalence is highly associated with the degree of airflow limitation [64]. The prevalence of pre-frailty is inversely associated with the severity of airflow limitation. The reason behind this is that people with severe disease transfer from a pre-frail to a frail state. COPD is closely related to frailty as both are associated with ageing, smoking, deregulated inflammation and endocrine dysfunction. Mechanisms of accelerated ageing and related inflammation in genetically susceptible individuals might underlie both COPD and frailty. Epigenetic alterations and altered intercellular communication are ageing mechanisms which are likely to be involved in lung diseases such as COPD and idiopathic pulmonary fibrosis (IPF) $[65,66]$. We need to better understand which mechanisms drive frailty in patients with COPD, as frailty reduces quality of life [67] and increases hospitalisations, hospital readmissions [68] and in-hospital mortality [69] in these patients.

\section{Rehabilitative interventions to reverse frailty (M. Maddocks, UK)}

The final speaker in this symposium discussed rehabilitative interventions in frailty. M. Maddocks provided an overview of the effectiveness and ease of implementation of a range of possible interventions regarding frailty. Exercise-based interventions appear to be an overall best solution, comprising both easy implementation and demonstrated effectiveness [70]. In a study by MADDOCKs et al. [71], results showed that overall rehabilitation in patients with COPD led to a shift away from frailty and towards a more robust state. Proportionally, more frail patients experienced deterioration in their condition or hospital admission during the programme. Frailty was also a strong independent predictor of noncompletion. In this regard, participants had to overcome unpredictable disruption to participation: some maintained determination despite disruption, others were supported by rapport and flexibility of services, but for some, pulmonary rehabilitation was no longer seen as a good fit [72]. A list of approaches on how to adapt rehabilitation programmes for frailty was given, comprising building trusting relationships, shared 
understanding of priorities, individualised content to match priorities, capacity to address multidimensional losses and flexibility in service delivery [73].

Take-home messages

- There exist several definitions of frailty and multiple assessment tools to evaluate it.

- Muscle satellite cell content is not significantly different between patients with COPD and healthy age-matched controls.

- The distance from a muscle satellite cell to its nearest capillaries is significantly greater in patients with COPD compared with healthy age-matched controls.

- Frailty may be acquired during illness and hospitalisation. It is an independent risk factor for poor outcome. It may occur with or separate from disability and cognitive impairment. Frailty may be uniquely amenable to rehabilitation and represent a novel opportunity for intervention.

- Frailty is common $(>10 \%)$ in patients with COPD and is the strongest determinant of survival in COPD. Frailty and COPD share inflammatory mechanisms.

- Frailty can be prevented and reversed by rehabilitation. Exercise remains a powerful intervention for people with respiratory disease and frailty.

- Pulmonary rehabilitation needs to "flex" to ensure a good fit for some of this group, without compromising quality.

\section{Group 9.03: Nurses}

Symposium: Fear and anxiety, psychological wellbeing and prevention of psychological distress in chronic lung diseases

This symposium aimed to identify psychological symptoms in chronic respiratory patients and provide novel treatment approaches that focus on psychological wellbeing and prevention of psychological distress. Four respiratory expert speakers provided an update on the latest knowledge about psychological comorbidities and its management. In addition, the testimony of a patient with a diagnosis of IPF was included.

The patient's perspective (R. Flewett, UK)

This symposium presented the opportunity to voice the experience of a patient with IPF giving advice for healthcare professionals. R. Flewett was diagnosed with IPF at the age of 53 years and given a life expectancy of 3-5 years. "I just felt so alone and so desperate at that point, having no one to turn to for help", R. Flewett said after diagnosis. Frustrated by previous medical experiences, fears and changing moods, R. Flewett provided four points of advice to healthcare professionals: 1) encourage communication, 2) reassure the patient that it is OK to feel sad, 3) put them in touch with other chronic patients and 3) promote exercise but clarify the dangers of overexertion. R. Flewett highlighted the nurse-patient relationship, which enables patients' requirements to be identified in a confidential environment, and the perception of the patient's family, the caregiver mainly. R. Flewett emphasised that "sometimes it is the simplest gestures that can help to unlock suppressed emotions".

\section{Disease-specific fears in COPD (T. Rejinders, Belgium)}

Anxiety is the main psychological comorbidity, being observed in $10-55 \%$ of patients with COPD [74]. Multiple studies have suggested that disease-specific fears of patients with COPD may aid or interfere with participation in pulmonary rehabilitation and self-care $[75,76]$. The principal fears of these patients are dyspnoea perception and physical activity because of the increase in this symptom [77]. Patients showed a greater perception, but also greater neural processing of respiratory sensations, which emphasises the attention-demanding feature of these sensations [78]. Patients with COPD with higher levels of dyspnoea-specific fear show increased attention to respiratory sensations and decreased physical activity. T. Rejinders demonstrated that high levels of disease-specific fears independent of general anxiety were associated with poor outcomes of pulmonary rehabilitation in patients with COPD [79]. Therefore, dyspnoea-specific fears in patients with COPD should be identified as early as possible to improve the effects of pulmonary rehabilitation.

Nurse-led cognitive behavioural therapy in COPD (K. Heslop-Marshall, UK)

Dyspnoea is the main symptom in patients with COPD. However, anxiety and depression have a significant impact despite not being evaluated and treated properly. Thus, the key elements of self-management in patients with COPD are: 1) mental health, 2) physical activity and 3) addressing physical symptoms. K. Heslop-Marshall highlighted the importance of addressing psychological comorbidities and how cognitive behavioural therapy (CBT) delivered by respiratory nurses can help patients with COPD. CBT is an evidence-based intervention for anxiety and depression in the general 
population [80]. This intervention was found to decrease anxiety levels and reduce resources use and hospital admission in patients with COPD, being a cost-effective intervention [81].

\section{Expressing empathy in 15 min or less: challenges for clinicians (D. Reynolds-Sandford, Australia)}

Empathy is the ability to understand and share the feelings of another person, discovering what that person needs, and then giving that [82]. Empathy is a skill anyone can develop. Practising empathy involves: 1) understanding and responding to the suffering of others, 2) challenging prejudices and preconceived ideas, 3) practising active listening, and 4) speaking up [83]. Healthcare interventions provided with empathy showed many benefits, including enhanced patient's experiences, adherence to treatment recommendations, better clinical outcomes and fewer malpractice claims [84]. Nevertheless, there is a negative part with a higher psychological cost to healthcare professionals. "Vicarious trauma" refers to the professional's continuous emotional engagement with patients' problems that creates cognitive distortions and changes in core belief systems within the professionals [85]. It is important that healthcare professionals understand the symptoms associated with this phenomenon to identify, prevent and/or minimise their effects.

\section{Integrating early palliative care for patients with IPF and their caregivers (K. Lindell, USA)}

IPF is associated with variable disease course, tremendous symptom burden and poor quality of life [86]. Palliative care should be initiated early in the illness course according to the individual patient's needs, preferences and culture to overcome the symptom burden and poor quality of life [87]. SUPPORT is an intervention aimed to provide education about seven key elements in IPF patients: (S) symptom management, $(\mathrm{U})$ understanding their disease, $(\mathrm{P})$ pulmonary rehabilitation, $(\mathrm{P})$ palliative care, $(\mathrm{O})$ oxygen therapy, (R) research opportunities and $(\mathrm{T})$ transplantation. The final objectives of SUPPORT were understanding the illness, caring for the patient, providing information for the carer, and planning for the future to improve symptom burden and quality of life [88].

Take-home messages

- The main fears of patients with COPD are dyspnoea perception and physical activity.

- CBT is a cost-effective intervention to decrease anxiety levels in patients with COPD.

- Empathy is a skill that healthcare professionals can develop to enhance patients' experiences and clinical outcomes.

- Palliative care should be initiated early in the illness course to overcome the symptom burden and poor quality of life.

\section{Oral presentation: Novel insights into nursing interventions for managing patients with asthma} and $C O P D$

This virtual oral presentation session provided an overview of state-of-the-art advances in nursing care related to respiratory conditions by focusing on three main themes: treatment adherence (three presentations), self-management (two presentations) and patients' perspectives (two presentations).

\section{Treatment adherence}

Reasons for inhaled corticosteroid nonadherence disclosed by African American adults during primary care visits for uncontrolled asthma (M. George, USA)

M. George's group aimed to identify reasons for inhaled corticosteroid (ICS) nonadherence by African American adults with asthma during primary care visits. Using grounded theory, three themes emerged from the analysis of audio recordings of 80 visits for uncontrolled asthma within African American adults: 1) personal misconceptions, 2) management confusions and 3) external influence. Findings suggest that primary care providers should assess patients' knowledge, beliefs and suspicions, and consider patient-centred strategies to improve ICS adherence (e.g. shared decision making and patient engagement).

\section{Treatment adherence among adults with asthma: a report from the OLIN asthma cohort}

(S.W. Lindmark, Sweden)

Similarly to the study to M. George, in the study presented by S.W. Lindmark, 1006 adults with asthma were followed up to study pharmacological treatment adherence in northern Sweden. Results showed that 579 participants used asthma treatment during the last year and also that that three out of five had high adherence according to the MARS-5 medication adherence report scale. Forgetfulness was the most common reason for nonadherence and males had poorer adherence than females. The authors of the study emphasised the importance of healthcare professionals to monitor adherence and reasons for nonadherence among patients with asthma. 
Study to evaluate satisfaction with the inhalation device used by patients with asthma or COPD and the association with adherence and disease control (D. Diaz-Perez, Spain)

D. Diaz-Perez presented a multicentre observational cross-sectional study evaluating patients' satisfaction with the inhalation device used and its association with adherence and disease control. The study included 400 participants with asthma and COPD, and results showed a high degree of satisfaction, with no difference among device types [89]. Furthermore, having a higher level of education was associated with a higher level of satisfaction. Findings suggest that shared decision making between patients and healthcare professionals is a key factor for the acceptance of the inhalation device.

\section{Self-management}

Ecare-COPD: a massive open online course to empower nurses to enhance self-management skills in patients with COPD (M. Padilha, Portugal)

Ecare-COPD was presented by $M$. Padilha as an online training programme for nurses to promote self-management in people with COPD. This e-learning tool was available in three levels (nursing students, general nurses and specialist nurses) with positive feedback from users in terms of usefulness, easiness and intention to use similar online courses.

An exploratory qualitative study of patients' and healthcare professionals' views on self-management in bronchiectasis (C. Kelly, UK)

C. Kelly's group conducted a study to explore preferences of self-management in adults with bronchiectasis and healthcare professionals involved in their care. Participants were recruited from three sites and participated in three focus groups (17 patients) and 11 one-to-one interviews with healthcare professionals. Findings revealed four themes: 1) what is self-management, 2) the purpose and impact of self-management, 3) barriers to self-management, and 4) enablers of self-management. The authors of the study concluded that both groups were positive about self-management, but some barriers were identified. Specialised healthcare professionals were recognised as precursors to effective self-management.

Patients' perspectives

Patients' perspectives on point-of-care diagnostics and treatment by emergency medical technicians in acute COPD exacerbations: a qualitative study (H.M. Christensen, Denmark)

In Demark, H.M. Christensen's group explored patients' perspectives on point-of-care diagnostics and treatment by emergency medical technicians in acute COPD exacerbations. The group conducted 19 qualitative semistructured interviews which revealed that patients valued the experience and safety of the ambulance staff using technical equipment and treatment of dyspnoea. Additionally, patients felt confident when general practitioners followed up the treatment initiated during the emergency episodes at home.

An exploration of dyspnoea and determinants in patients with COPD (E.M. Nerheim, Norway) E.M. Nerheim's group examined how physical and affective dimensions of dyspnoea were associated in 203 patients with COPD attending a 4-week inpatient pulmonary rehabilitation programme. According to the Hospital Anxiety and Depression Scales (HADS), results showed that patients with greater dyspnoea severity reported a higher degree of anxiety (HADS-A; B: 0.97, $\mathrm{p}<0.001)$ ) and depression (HADS-D; B: $0.55, \mathrm{p}=0.002$ ). The group concluded that an individual approach is needed to explore physical and affective dimensions of dyspnoea.

Take-home messages

- Respiratory professionals should monitor treatment adherence and reasons for nonadherence in patients with respiratory conditions.

- Patient engagement and shared decision making between clinicians and patients might be useful to improve treatment adherence in respiratory care.

- Training and specialised knowledge in respiratory care are important to implement effective self-management interventions.

- Patients' perspectives need to be considered during assessment and treatment in respiratory care.

\section{Concluding remarks}

This article highlights some of the most memorable sessions during the virtual ERS International Congress 2020, and provides readers with valuable insight into the latest scientific data and emerging areas affecting allied respiratory professionals' clinical practice. The authors wish to inspire readers' enthusiasm to appreciate the value of staying up to date in their field of interest. A new scientific group was created this year in the Allied Health Professionals Assembly, Group 9.04: Psychologists and Behavioural 
Scientists; therefore, we believe that next year there will be even more interesting sessions to share the latest scientific data. We hope to see you all in Barcelona in 2021 for the next highly successful ERS International Congress.

Conflict of interest: None declared.

Support statement: J. De Brandt is funded by the Flemish government. The research of FWO Aspirant J. De Brandt is sponsored by FWO grant number 11B4718N. J. Cruz acknowledges the support of the Center for Innovative Care and Health Technology (ciTechCare) of the Polytechnic of Leiria, funded by Portuguese national funds provided by Fundação para a Ciência e Tecnologia (FCT) (UIDB/05704/2020 and UIDP/05704/2020).

\section{References}

1 Coss P, King G, McLaughlin A, et al. 30 Early effect of continuous positive airway pressure therapy on left atrial mechanics in patients with obstructive sleep apnoea: assessment by conventional and two-dimensional speckle-tracking echocardiography. Heart 2018; 104: Suppl. 7, A22-A23.

2 American Thoracic Society/American College of Chest Physicians. ATS/ACCP statement on cardiopulmonary exercise testing. Am J Respir Crit Care Med 2003; 167: 211-277.

3 Radtke T, Crook S, Kaltsakas G, et al. ERS statement on standardisation of cardiopulmonary exercise testing in chronic lung diseases. Eur Respir Rev 2019; 28: 180101

4 Cooper DM, Weiler-Ravell D, Whipp BJ, et al. Aerobic parameters of exercise as a function of body size during growth in children. J Appl Physiol Respir Environ Exerc Physiol 1984; 56: 628-634.

5 Blanchard J, Blais S, Chetaille P, et al. New reference values for cardiopulmonary exercise testing in children. Med Sci Sports Exerc 2018; 50: 1125-1133.

6 Bongers B, van Brussel M, Hulzebos EH, et al. Pediatric Norms for Cardiopulmonary Exercise Testing in Relation to Sex and Age. s-Hertogenbosch, Uitgeverij BOXpress, 2014

7 Das N, Verstraete K, Stanojevic S, et al. Deep learning algorithm helps to standardise ATS/ERS spirometric acceptability and usability criteria. Eur Respir J 2020; 56: 2000603.

8 Miller MR, Hankinson J, Brusasco V, et al. Standardisation of spirometry. Eur Respir J 2005; 26: 319-338.

9 Graham BL, Steenbruggen I, Miller MR, et al. Standardization of Spirometry 2019 Update. An Official American Thoracic Society and European Respiratory Society Technical Statement. Am J Respir Crit Care Med 2019; 200: e70-e88.

10 Stanojevic S, Graham BL, Cooper BG, et al. Official ERS technical standards: Global Lung Function Initiative reference values for the carbon monoxide transfer factor for Caucasians. Eur Respir J 2017; 50: 1700010.

11 Pellegrino R, Viegi G, Brusasco V, et al. Interpretative strategies for lung function tests. Eur Respir J 2005; 26 948-968.

12 Quanjer PH, Pretto JJ, Brazzale DJ, et al. Grading the severity of airways obstruction: new wine in new bottles. Eur Respir J 2014; 43: 505-512.

13 Holguin F, Cardet JC, Chung KF, et al. Management of severe asthma: a European Respiratory Society/American Thoracic Society guideline. Eur Respir J 2020; 55: 1900588

14 Asthma UK. Smart Asthma: Real World Implementation of Connected Devices in the UK to Reduce Asthma Attacks. London, Asthma UK, 2017.

15 Kikidis D, Konstantinos V, Tzovaras D, et al. The digital asthma patient: the history and future of inhaler based health monitoring devices. J Aerosol Med Pulm Drug Deliv 2016; 29: 219-232.

16 Bonini M, Usmani OS. Novel methods for device and adherence monitoring in asthma. Curr Opin Pulm Med 2018; 24: 63-69.

17 Sulaiman I, Seheult J, Sadasivuni N, et al. The impact of common inhaler errors on drug delivery: investigating critical errors with a dry powder inhaler. J Aerosol Med Pulm Drug Deliv 2017; 30: 247-255.

18 Chan AH, Harrison J, Black PN, et al. Using electronic monitoring devices to measure inhaler adherence: a practical guide for clinicians. J Allergy Clin Immunol Pract 2015; 3: 335-49.

19 Hui CY, Walton R, McKinstry B, et al. The use of mobile applications to support self-management for people with asthma: a systematic review of controlled studies to identify features associated with clinical effectiveness and adherence. J Am Med Inform Assoc 2017; 24: 619-632.

20 Simpson AJ, Honkoop PJ, Kennington E, et al. Perspectives of patients and healthcare professionals on mHealth for asthma self-management. Eur Respir J 2017; 49: 1601966.

21 Mishlanov V, Chuchalin A, Chereshnev V, et al. Scope and new horizons for implementation of m-Health/ e-Health services in pulmonology in 2019. Monaldi Arch Chest Dis 2019; 89: 1112.

22 Hwang EJ, Park S, Jin KN, et al. Development and validation of a deep learning-based automatic detection algorithm for active pulmonary tuberculosis on chest radiographs. Clin Infect Dis 2019; 69: 739-747.

23 Huang L, Han R, Ai T, et al. Serial quantitative chest CT assessment of COVID-19: deep-learning approach. Radiol Cardiothorac Imaging 2020; 2: e200075.

24 Chen P-HC, Gadepalli K, MacDonald R, et al. An augmented reality microscope with real-time artificial intelligence integration for cancer diagnosis. Nat Med 2019; 25: 1453-1457.

25 Xiong Y, Ba X, Hou A, et al. Automatic detection of Mycobacterium tuberculosis using artificial intelligence. J Thorac Dis 2018; 10: 1936-1940.

26 Topalovic M, Das N, Burgel PR, et al. Artificial intelligence outperforms pulmonologists in the interpretation of pulmonary function tests. Eur Respir J 2019; 53: 1801660.

27 Shakhmametova G, Zulkarneev R, Evgrafov A. Clinical decision support system for the respiratory diseases diagnosis. 2019. www.atlantis-press.com/article/125908965.pdf Date last accessed: December 12, 2020.

28 Harrer S, Shah P, Antony B, et al. Artificial intelligence for clinical trial design. Trends Pharmacol Sci 2019; 40: 577-591.

29 Taylor K, Properzi F, Cruz M. Intelligent clinical trials: transforming through AI-enabled engagement. 2020. www2.deloitte.com/us/en/insights/industry/life-sciences/artificial-intelligence-in-clinical-trials.html Date last accessed: December 12, 2020. 

and sensitivity in three search tools for qualitative systematic reviews. BMC Health Serv Res 2014; 14. 579.

31 McCool FD, Rosen MJ. Nonpharmacologic airway clearance therapies: ACCP evidence-based clinical practice guidelines. Chest 2006; 129: 1 Suppl., 250s-259s.

32 Volsko TA. Airway clearance therapy: finding the evidence. Respir Care 2013; 58: 1669-1678.

33 Polverino E, Goeminne PC, McDonnell MJ, et al. European Respiratory Society guidelines for the management of adult bronchiectasis. Eur Respir J 2017; 50: 1700629.

34 Wilson LM, Morrison L, Robinson KA. Airway clearance techniques for cystic fibrosis: an overview of Cochrane systematic reviews. Cochrane Database Syst Rev 2019; 1: CD011231.

35 McIlwaine M, Bradley J, Elborn JS, et al. Personalising airway clearance in chronic lung disease. Eur Respir Rev 2017; 26: 160086.

36 Basavaraj A, Choate R, Addrizzo-Harris D, et al. Airway clearance techniques in bronchiectasis: analysis from the United States Bronchiectasis and Non-TB Mycobacteria Research Registry. Chest 2020; 158: 1376-1384.

37 Franks LJ, Walsh JR, Hall K, et al. Measuring airway clearance outcomes in bronchiectasis: a review. Eur Respir Rev 2020; 29: 190161.

38 Osadnik CR, McDonald CF, Jones AP, et al. Airway clearance techniques for chronic obstructive pulmonary disease. Cochrane Database Syst Rev 2012; 3: CD008328.

39 Osadnik CR, McDonald CF, Miller BR, et al. The effect of positive expiratory pressure (PEP) therapy on symptoms, quality of life and incidence of re-exacerbation in patients with acute exacerbations of chronic obstructive pulmonary disease: a multicentre, randomised controlled trial. Thorax 2014; 69: 137-143.

40 Lee AL, Burge AT, Holland AE. Airway clearance techniques for bronchiectasis. Cochrane Database Syst Rev 2015 11: CD008351

41 Muñoz G, de Gracia J, Buxó M, et al. Long-term benefits of airway clearance in bronchiectasis: a randomised placebo-controlled trial. Eur Respir J 2018; 51: 1701926.

42 Warnock L, Gates A. Chest physiotherapy compared to no chest physiotherapy for cystic fibrosis. Cochrane Database Syst Rev 2015; 12: CD001401.

43 Spinou A, Chalmers JD. Respiratory physiotherapy in the bronchiectasis guidelines: is there a loud voice we are yet to hear? Eur Respir J 2019; 54: 1901610.

44 Morley JE, Vellas B, van Kan GA, et al. Frailty consensus: a call to action. J Am Med Dir Assoc 2013; 14: 392-397.

45 Fried LP, Tangen CM, Walston J, et al. Frailty in older adults: evidence for a phenotype. J Gerontol A Biol Sci Med Sci 2001; 56: M146-M156.

46 Mitnitski AB, Mogilner AJ, Rockwood K. Accumulation of deficits as a proxy measure of aging ScientificWorldJournal 2001; 1: 321027.

47 Rockwood K, Song X, MacKnight C, et al. A global clinical measure of fitness and frailty in elderly people. CMAJ 2005; 173: 489-495.

48 Buta BJ, Walston JD, Godino JG, et al. Frailty assessment instruments: systematic characterization of the uses and contexts of highly-cited instruments. Ageing Res Rev 2016; 26: 53-61.

49 Ellis G, Gardner M, Tsiachristas A, et al. Comprehensive geriatric assessment for older adults admitted to hospital. Cochrane Database Syst Rev 2017; 9: CD006211.

50 World Health Organization. Integrated care for older people: guidelines on community-level interventions to manage declines in intrinsic capacity. 2017. www.apps.who.int/iris/handle/10665/258981 Date last accessed: December 12, 2020.

51 Verdijk LB, Snijders T, Drost M, et al. Satellite cells in human skeletal muscle; from birth to old age. Age 2014; 36 545-547.

52 Thériault M-E, Paré MÈ, Lemire BB, et al. Regenerative defect in vastus lateralis muscle of patients with chronic obstructive pulmonary disease. Respir Res 2014; 15: 35-35.

53 Bentzinger CF, von Maltzahn J, Rudnicki MA. Extrinsic regulation of satellite cell specification. Stem Cell Res Ther 2010; 1: 27.

54 Nederveen JP, Joanisse S, Snijders T, et al. Skeletal muscle satellite cells are located at a closer proximity to capillaries in healthy young compared with older men. J Cachexia Sarcopenia Muscle 2016; 7: 547-554.

55 Bagshaw SM, Stelfox HT, McDermid RC, et al. Association between frailty and short- and long-term outcomes among critically ill patients: a multicentre prospective cohort study. CMAJ 2014; 186: E95-E102.

56 Brummel NE, Girard TD, Pandharipande PP, et al. Prevalence and course of frailty in survivors of critical illness. Crit Care Med 2020; 48: 1419-1426.

57 Herridge MS, Cheung AM, Tansey CM, et al. One-year outcomes in survivors of the acute respiratory distress syndrome. N Engl J Med 2003; 348: 683-693.

58 Levine S, Nguyen T, Taylor N, et al. Rapid disuse atrophy of diaphragm fibers in mechanically ventilated humans. N Engl J Med 2008; 358: 1327-1335.

59 Puthucheary ZA, Rawal J, McPhail M, et al. Acute skeletal muscle wasting in critical illness. JAMA 2013; 310: 1591-1600.

60 Ferrante LE, Pisani MA, Murphy TE, et al. Functional trajectories among older persons before and after critical illness. JAMA Intern Med 2015; 175: 523-529.

61 Hopkins RO, Weaver LK, Pope D, et al. Neuropsychological sequelae and impaired health status in survivors of severe acute respiratory distress syndrome. Am J Respir Crit Care Med 1999; 160: 50-56.

62 Pandharipande PP, Girard TD, Jackson JC, et al. Long-term cognitive impairment after critical illness. N Engl J Med 2013; 369: 1306-1316.

63 Dowdy DW, Bienvenu OJ, Dinglas VD, et al. Are intensive care factors associated with depressive symptoms 6 months after acute lung injury? Crit Care Med 2009; 37: 1702-1707.

64 Lahousse L, Ziere G, Verlinden VJ, et al. Risk of frailty in elderly with COPD: a population-based study. J Gerontol A Biol Sci Med Sci 2016; 71: 689-695.

65 Lahousse L, Moyse E, Krantic S, et al. Understanding age-related diseases: report of the 2015 Ageing Summit. Eur Respir J 2016; 47: 5-9.

66 López-Otín C, Blasco MA, Partridge L, et al. The hallmarks of aging. Cell 2013; 153: 1194-1217. 
Kennedy CC, Novotny PJ, LeBrasseur NK, et al. Frailty and clinical outcomes in chronic obstructive pulmonary disease. Ann Am Thorac Soc 2019; 16: 217-224.

68 Bernabeu-Mora R, García-Guillamón G, Valera-Novella E, et al. Frailty is a predictive factor of readmission within 90 days of hospitalization for acute exacerbations of chronic obstructive pulmonary disease: a longitudinal study. Ther Adv Respir Dis 2017; 11: 383-392.

69 Warwick M, Fernando SM, Aaron SD, et al. Outcomes and resource utilization among patients admitted to the intensive care unit following acute exacerbation of chronic obstructive pulmonary disease. J Intensive Care Med 2020; in press [https://doi.org/10.1177/0885066620944865].

70 Travers J, Romero-Ortuno R, Bailey J, et al. Delaying and reversing frailty: a systematic review of primary care interventions. Br J Gen Pract 2019; 69: e61-e69.

71 Maddocks M, Kon SS, Canavan JL, et al. Physical frailty and pulmonary rehabilitation in COPD: a prospective cohort study. Thorax 2016; 71: 988-995.

72 Brighton LJ, Bristowe K, Bayly J, et al. Experiences of pulmonary rehabilitation in people living with chronic obstructive pulmonary disease and frailty. a qualitative interview study. Ann Am Thorac Soc 2020; 17: 1213-1221.

73 Donaldson AV, Maddocks M, Martolini D, et al. Muscle function in COPD: a complex interplay. Int J Chron Obstruct Pulmon Dis 2012; 7: 523-535.

74 Willgoss TG, Yohannes AM. Anxiety disorders in patients with COPD: a systematic review. Respir Care 2013; 58: $858-866$.

75 Wortz K, Cade A, Menard JR, et al. A qualitative study of patients' goals and expectations for self-management of COPD. Prim Care Respir J 2012; 21: 384-391.

76 De Peuter S, Janssens T, Van Diest I, et al. Dyspnea-related anxiety: the Dutch version of the Breathlessness Beliefs Questionnaire. Chron Respir Dis 2011; 8: 11-19.

77 Vardar-Yagli N, Calik-Kutukcu E, Saglam M, et al. The relationship between fear of movement, pain and fatigue severity, dyspnea level and comorbidities in patients with chronic obstructive pulmonary disease. Disabil Rehabil 2019; 41: 2159-2163.

78 Reijnders T, Troosters T, Janssens W, et al. Brain activations to dyspnea in patients with COPD. Front Physiol 2020; $11: 7$.

79 Reijnders T, Schuler M, Wittmann M, et al. The impact of disease-specific fears on outcome measures of pulmonary rehabilitation in patients with COPD. Respir Med 2019; 146: 87-95.

80 Hynninen MJ, Bjerke N, Pallesen S, et al. A randomized controlled trial of cognitive behavioral therapy for anxiety and depression in COPD. Respir Med 2010; 104: 986-994.

81 Heslop-Marshall K, Baker C, Carrick-Sen D, et al. Randomised controlled trial of cognitive behavioural therapy in COPD. ERJ Open Res 2018; 4: 00094-2018.

82 Elliott R, Bohart AC, Watson JC, et al. Empathy. Psychotherapy 2011; 48: 43-49.

83 Riess H. The science of empathy. J Patient Exp 2017; 4: 74-77.

84 Riess H, Kelley JM, Bailey RW, et al. Empathy training for resident physicians: a randomized controlled trial of a neuroscience-informed curriculum. J Gen Intern Med 2012; 27: 1280-1286.

85 Pearlman LA, Mac Ian PS. Vicarious traumatization: an empirical study of the effects of trauma work on trauma therapists. Prof Psychol Res Pract 1995; 26: 558-565.

86 Carvajalino S, Reigada C, Johnson MJ, et al. Symptom prevalence of patients with fibrotic interstitial lung disease: a systematic literature review. BMC Pulm Med 2018; 18: 78.

87 Kreuter M, Bendstrup E, Russell AM, et al. Palliative care in interstitial lung disease: living well. Lancet Respir Med 2017; 5: 968-980.

88 Lindell KO, Nouraie M, Klesen MJ, et al. Randomised clinical trial of an early palliative care intervention (SUPPORT) for patients with idiopathic pulmonary fibrosis (IPF) and their caregivers: protocol and key design considerations. BMJ Open Respir Res 2018; 5: e000272.

89 Donaire JG, Pérez DD, Hernández C, et al. Study to evaluate satisfaction with the inhalation device used by patients with asthma or chronic obstructive pulmonary disease and the association with adherence and disease control. J Aerosol Med Pulm Drug Deliv 2020; 33: 153-160. 